\title{
Le novità del concilio Vaticano II sul ministero ordinato
}

\author{
The Innovations of the Second Vatican Council \\ on Ordained Ministry
}

DARIO VITALI

\author{
Pontificia Univesidad Gregoriana (Roma) \\ vitali@unigre.it \\ Fecha de recepción: 22/7/2021 \\ Fecha de aceptación: 20/8/2021 \\ https://doi.org/10.52039/seminarios.v66i228.442
}

SOMMARIO: Il presente contributo studia le novità sul ministero ordinato emerse al concilio Vaticano II. Dal punto di vista della storia del dogma, il concilio ha costituito un passaggio importante. Attraverso un confronto con il decreto del concilio di Trento sul sacramento dell'Ordine, sono stati individuati i nuclei più rilevanti di novità: il sacerdozio di Cristo, ricompreso nella prospettiva del mistero pasquale ( $S C$ 7); il sacerdozio comune dei fedeli e la sua relazione con il sacerdozio ministeriale ( $L G 10)$; la dottrina sull'episcopato come sacramento ( $L G 21)$ e la conseguente ricomposizione del quadro degli ordini: episcopato, presbiterato, diaconato ( $L G$ 28-29); la dottrina dei tria munera come schema interpretativo per comprendere l'esercizio del ministero ordinato. L'esame dei testi conciliari permette di comprendere come il mutamento del modello ecclesiologico, voluto dal concilio, richieda di sviluppare un modello di ministero ordinato coerente con i principi che fondano l'ecclesiologia del Vaticano II.

Parole ChiAVE: Sacramento dell'Ordine, Sacerdozio, Episcopato, Presbiterato, Diaconato.

ABSTRACT: This paper studies the innovations regarding the ordained ministry that emerged at the Second Vatican Council. From the point of view of the history of dogma, the Council was an important step. Through a comparison with the decree of the Council of Trent on the sacrament of Order, the most important elements of novelty have been identified: the priesthood of Christ, included in the perspective of the Paschal Mystery (SC 7); the common priesthood of the faithful and its relationship with ordained priesthood (LG 10); the doctrine on the episcopate as a sacrament (LG 21) and the consequent rearrangement of the pattern of orders: episcopacy, presbyterate, diaconate (LG 28-29); the doctrine of the tria munera as an interpretative template for understanding the exercise of the ordained ministry. An examination of the Council texts reveals how the change in the ecclesiological model desired by the Council requires the development of a model of ordained ministry consistent with the principles underlying the ecclesiology of Vatican II.

KEYWORDS: Sacrament of Order, Priesthood, Episcopate, Presbyterate, Diaconate. 
È ormai abituale evocare la novità quando si parla del concilio Vaticano II. Se questo non è sempre vero e spesso rasenta il luogo comune, vale senz'altro per il tema del ministero ordinato, sul quale il concilio è ripetutamente intervenuto. L'esame dei documenti conciliari mostrerà quali e quanti sono gli elementi di novità introdotti, che hanno profondamente inciso sul quadro tradizionale fissato dal concilio di Trento.

Elementi che - va detto subito - non hanno il carattere della sistematicità. Unicamente Presbyterorum ordinis è un testo organico; tuttavia non affronta l'intera questione, perché si limita a trattare la parte relativa a uno degli ordini. Peraltro, si tratta di un decreto di riforma - quindi non un documento di prima grandezza -, tuttavia costruito a partire da elementi formulati altrove e per altre ragioni che quelle del ministero.

Ciò nonostante, il fatto che l'impostazione tridentina del sacramento dell'Ordine identificasse il sacerdozio con il presbiterato ha determinato un problema non irrilevante di ermeneutica conciliare: i tentativi post-conciliari di ripensare il ministero ordinato hanno faticato a smarcarsi da tale identificazione. Gli autori hanno finito per concentrare ogni sforzo su Presbyterorum ordinis, quasi bastasse rideclinare il sacerdozio in termini di ministero per cogliere le novità del concilio e avviare il rinnovamento teologico del tema.

Per questo riveste grande importanza un approccio diacronico ai testi, per mostrare la genesi e le tappe di una evoluzione che aspetta ancora una sintesi compiuta. Il valore del presente contributo sta nella ricostruzione precisa di quello sviluppo, situando con precisione i singoli elementi di novità, a partire dai quali costruire una proposta teologico-sacramentale sul ministero ordinato in linea con in modello conciliare di Chiesa.

\section{Due MODELli A CONFRONTO}

Per individuare gli aspetti di novità del Vaticano II sul ministero ordinato, bisogna anzitutto rammentare il quadro dottrinale del concilio di Trento, che costituisce il termine di riferimento per misurare le differenze introdotte dal concilio. Differenze che si colgono già nell'approccio al tema, soprattutto quando si elegga Presbyterorum ordinis come misura di confronto: pastorale il decreto di riforma del Vaticano II; teologico e sacramentale il decreto di Trento. La questione di fondo è se alla diversità di approccio si accompagni anche una diversità di contenuti, e - in caso affermativo - se si tratti di novità effettive, o solo di diversa sensibilità nel declinare il tema del ministero ordinato. 
Il taglio che Trento dà alla questione si coglie già dal titolo del decreto: «Dottrina e canoni sul sacramento dell'Ordine» ${ }^{1}$. Il punto di vista è quello sacramentale, con una forte accentuazione apologetica: scopo del decreto è fissare «la vera dottrina cattolica sul sacramento dell'Ordine, a condanna degli errori del nostro tempo, stabilita e resa pubblica dal santo Concilio di Trento nella sessione settima $»^{2}$. Per legittimare tale dottrina, il concilio ha spostato l'attenzione dalla gerarchia ecclesiastica, contestata dai Riformatori come invenzione ecclesiastica, al sacerdozio. Per i teologi di Trento la legittimazione incontestabile della gerarchia sta nel legame che sussiste tra sacrificio e sacerdozio, che vale per ogni legge e vale, a maggior ragione, per la Nuova Legge: «Poiché nel Nuovo Testamento la Chiesa cattolica ha ricevuto dall'istituzione stessa del Signore il santo visibile sacrificio dell'Eucaristia, bisogna pure confessare che in essa esiste anche un nuovo sacerdozio visibile ed esteriore», istituito da Cristo stesso; è lui, infatti, che ha conferito «agli Apostoli e ai loro successori nel sacerdozio il potere di consacrare, di offrire e distribuire il suo corpo e il suo sangue, come pure di rimettere o non rimettere i peccati» ${ }^{3}$.

Quella descritta è la potestas ordinis, anche se il decreto volutamente non la cita, per non entrare in una disputa di scuola; piuttosto, assume i dati della Sacra Scrittura e della Tradizione, armonizzandoli con una visione sacrale del ministero. Così, nel capitolo 2 si legano i sette ordini - quattro minori, tre maggiori - in una sequenza che appare come una scala per ascendere al sacerdozio come al grado più alto in dignità nella Chiesa. Né poteva essere altrimenti, in quanto l'ordinazione sacerdotale configura il ministro a Cristo stesso: dopo essere stato introdotto nello stato clericale con la tonsura, egli ha ricevuto in successione gli ordini minori - l'ostiariato, l'esorcistato, il lettorato e l'accolitato - e a seguire il suddiaconato e il diaconato, tutti ordini «che in ragione del loro ufficio sono a servizio del sa-

1. Concilio di Trento, Dottrina e canoni sul sacramento dell'Ordine (15. 07. 1563): DH 1763-1778.

2. $D H 1763$. Il decreto, in realtà, fu approvato nella sessione $23^{a}$, il 15 luglio 1563 . Ma in quell'occasione i Padri si rifecero a quanto già precedentemente discusso a Bologna nel 1547 e a Trento nel 1552; cfr. Concilium Tridentinum, Diariorum, Epistularum, Tractatuum nova collectio, Friburgo 1901, vol. 6, 97-308; vol. 7, 375-489; 460-489; vol. 9, 38-41; 105107; 226-241.

3. Capitolo 1: «L'istituzione del sacerdozio del Nuovo Testamento»: $D H$ 1764. Il capitolo 1 riveste teologicamente il can. 1: «Se qualcuno dirà che nel Nuovo Testamento non vi è un sacerdozio visibile ed esteriore, o che non vi è alcun potere di consacrare e di offrire il vero corpo e sangue del Signore, di rimettere e di ritenere i peccati, ma il solo ufficio di predicare il Vangelo, o che quelli che non predicano, non sono assolutamente sacerdoti, sia anatema»: $D H 1171$. 
cerdozio», per accedere finalmente al sacerdozio, nel quale è costituito come alter Christus, nella capacità di offrire nella Messa il sacrificio di Cristo sulla croce ${ }^{4}$.

Chiarito il carattere sacramentale dell'Ordine, che deve essere considerato «realmente e propriamente uno dei sette sacramenti della santa Chiesa» ${ }^{5}$, il concilio si applica a chiarire le conseguenze dell'ordinazione sacerdotale ${ }^{6}$. La più sorprendente riguarda chi faccia parte di questo «ordine ecclesiastico»: «praecipue i vescovi, successori degli Apostoli, i quali, stabiliti dallo Spirito santo «a pascere la Chiesa di Dio», sono superiori ai presbiteri» ${ }^{7}$. L'affermazione, motivata sulla base delle funzioni che competono esclusivamente al vescovo, differisce dalla spiegazione offerta nel Catechismo Romano $^{8}$, dove si recupera esplicitamente la distinzione tra potestas ordinis e potestas iurisdictionis, l'una che «riguarda il vero Corpo di Cristo Signore nella sacrosanta Eucaristia», l'altra che «si esercita tutta sul Corpo mistico di Cristo», alla quale «spetta governare e regolare il popolo cristiano e dirigerlo alla eterna beatitudine celeste ${ }^{9}$. Sempre seguendo la logica ascendente dei gradi, dopo aver spiegato le funzioni degli ordini minori e maggiori, il Catechismo chiarisce che «l'Ordine sacerdotale, pur essendo unico, ha vari gradi di dignità e di potestà», evidentemente di giurisdizione: «il primo è di quelli che si chiamano semplicemente sacerdoti»; a seguire nomina i vescovi, gli arcivescovi, i patriarchi, per terminare la piramide gerarchica con il Romano Pontefice, che «per diritto divino è il più grande di tutti i vescovi» ${ }^{10}$.

4. È quanto precisa il decreto sul sacrificio della Messa (sessione 22a 17 settembre 1562): nel capitolo 1 il concilio dice che nell'Ultima Cena Cristo «volle lasciare alla sua Chiesa, sua amata sposa, un sacrificio visibile, (come esige l'umana natura), con cui venisse significato quello cruento che avrebbe offerto una volta per tutte sulla croce»: $D H 1740$; nel capitolo 2 precisa che «in questo divino sacrificio che si compie nella Messa è Cristo stesso ad essere contenuto e immolato in modo incruento, lui che si offerse una volta sola sull'altare della croce (cfr $E b$ 9, 14. 27s)»: $D H 1743$.

5. Capitolo 3: «L'Ordine è veramente un sacramento»: $D H 1766$.

6. Capitolo 4: «La gerarchia ecclesiastica e l’ordine»: $D H$ 1767-1770.

7. $\mathrm{DH} 1768$.

8. Il Cathechismo romano, o Catechismus ad parochos, è stato redatto da una commissione di teologi istituiti da Pio IV e pubblicato nel 1566, con il titolo Catechismus ex decreto Concilii Tridentini ad parochos Pii Viussu editus, Romae 1566, in duplice versione: originale italiano, coordinata dal crd. Carlo Borromeo, e traduzione latina, condotta da due latinisti di chiara fama come Aldo Manuzio e da Giulio Poggiani. Per il testo in italiano: Catechismo del concilio di Trento, Paoline, Roma 1961.

9. Catechismo del Concilio di Trento, parte, II, cap. VII, n. 6.

10. Catechismo, parte II, cap. VII, nn. 26-28. 


\section{IL VOCABOLARIO}

Dunque, la categoria che il concilio di Trento pone al centro della sua dottrina è quella di sacerdozio, che esprime l'essenza del sacramento dell'Ordine, conferito a chi appartiene allo stato clericale; il Catechismo parla anche di ministri, ma il termine non risulta determinante. Questo ventaglio di termini ritorna anche nel Vaticano II: nei vari testi conciliari si ritrovano gli stessi vocaboli. Ma il campo semantico si allarga a dismisura con altri termini come munus, officium e ministerium, come pure attraverso un richiamo insistente ai singoli ordini e a chi li riceve: il vescovo, il presbitero, il diacono. Peraltro, la distribuzione dei termini non è omogenea, e non di rado risponde a logiche interne ad ogni specifico documento. Tuttavia, la difficoltà di recensire il vocabolario aiuta a capire la direzione voluta dal concilio in questo ambito ${ }^{11}$.

Nei documenti conciliari si incontrano i termini più tipici del vocabolario tridentino: quelli legati al sacerdozio ${ }^{12}$, ma anche quelli propri dello stato clericale $^{13}$, o della funzione ${ }^{14}$. Accanto a questi, però, emergono con prepotenza termini come minister/ministerium ${ }^{15}$, officium ${ }^{16}$, servitium ${ }^{17}$ e soprattutto $m u$ $n u s^{18}$, legato allo schema dei tria munera, che costituisce una delle novità più rilevanti del concilio non solo sul ministero ordinato, ma sulla partecipazione di tutti alla vita e alla missione della Chiesa. D'altra parte, una delle peculiarità del vocabolario conciliare sul ministero ordinato è quella di insistere

11. Per un esame dettagliato: X. Ochoa, Index verborum Concilii Vaticani II, Roma 1967.

12. Sacerdos ricorre 129 volte, di cui 24 in $S C, 16$ in $L G, 25$ in $C D, 11$ in $A G ; 10$ in $O T$, 31 in PO; 58 volte l'aggettivo sacerdotalis, di cui 7 in $L G, 20$ in $O T$ e 16 in $P O ; 34$ volte il sostantivo sacerdotium, di cui soltanto 2 in $S C, 13$ in $L G, 7$ in $O T$ e 15 in $P O$.

13. Clericus ricorre 33 volte, di cui 9 in $S C$, soltanto 2 in $L G$, 6 in $C D$ e 1 in $P O$; 3 ricorrenze dell'aggettivo clericalis. Clerus ricorre 29 volte, di cui 10 in $A G$, in rapporto al clero indigeno.

14. Hierarchia ricorre 36 volte, con sorprendente densità in Apostolicam Actuositatem: 16 ricorrenze; 29 volte l'aggettivo hierarchicus.

15. Minister/ministerialis ricorre 49 volte, di cui 5 in $S C ; 3$ in $O E ; 1$ in $C D ; 3$ in $O T ; 2$ in $D V ; 7$ in $A G$; 15 in $P O$; ministerium ricorre 149 volte, di cui: 7 in $S C ; 23$ in $L G ; 1$ in $O E ; 3$ in $U R$; 12 in $C D ; 7$ in $P C$; 11 in $O T$; 3 in $G E$; 3 in $D V ; 9$ in $A A ; 17$ in $A G ; 48$ in $P O ; 5$ in $G S$.

16. Officium ricorre 247 volte, di cui 37 in $S C ; 19$ in $I M ; 28$ in $L G ; 1$ in Nep; 2 in $O E$; 1 in $U R$; 26 in $C D ; 4$ in $P C ; 17$ in GE; 14 in $A A ; 15$ in $D H ; 12$ in $A G ; 21$ in $P O ; 41$ in GS. Va rammentato che il termine si applica anche ad ambiti diversi da quello del ministero ordinato, come appare evidente da $S C$ o da GE $o$ da GS.

17. Servitium ricorre 80 volte: le citazioni che rientrano nel campo del ministero ordinato sono soprattutto quelle di $L G$ (12 volte), $C D$ (4 volte) e $P O$ (11 volte).

18. Munus ricorre 255 volte, di cui 13 in SC; 5 in IM; 50 in $L G ; 6$ in Nep; 3 in $O E$; 6 in $U R ; 43$ in $C D ; 11$ in $P C$; 4 in $O T ; 12$ in GE; 1 in NE; 1 in $D V ; 12$ in $A A ; 2$ in $D H ; 19$ in $A G$; 22 in $P O$; 46 in GS. 
sulle figure - vescovi ${ }^{19}$, presbiteri $^{20}$, diaconi ${ }^{21}-$, che inquadra di preferenza come ordo $^{22}$ piuttosto che come gradus del sacramento dell'Ordine.

Se questo è il quadro delle ricorrenze, sarebbe presuntuoso - e pretestuoso - voler dedurre le novità del concilio sul ministero ordinato sulla base del solo vocabolario. L'insufficienza di una valutazione su base statistica è subito evidente, perché non si dà una costante nell'uso dei termini, né si può cogliere la decisione del Vaticano II di ricusare un termine in netto favore di un altro. L'esempio più evidente è il termine ministerium, spesso indicato come la cifra della novità conciliare rispetto alla caratterizzazione sacrale del sacerdozio, proposta dal concilio di Trento. Si darebbe per dimostrata tale volontà se il concilio sostituisse in modo sistematico sacerdos/sacerdotium/sacerdotalis con minister/ministerium/ministerialis. Ora, se è vero che il termine ministerium ricorre ben 149 volte, a fronte delle sole 34 ricorrenze di sacerdotium, il peso delle citazioni si rovescia quando si consideri non più la funzione in astratto, ma la persona che la svolge - il minister rispetto al sacerdos - e l'aggettivo che la qualifica: ministerialis rispetto a sacerdotalis: non solo sacerdos/sacerdotalis ricorre 188 volte, contro le 49 di minister/ministerialis, ma non poche volte ministerialis è qualificativo di sacerdotium ${ }^{23}$ !

Va certamente riconosciuta una concentrazione del termine ministerium in Presbyterorum ordinis. Questo può certamente significare che il concilio ha adottato tale chiave interpretativa soprattutto per ripensare il presbiterato. Ma non bisogna dimenticare che si tratta di un solo ordine e non dell'intero ministero ordinato, che consta di tre ordini, riarticolati dal concilio con scelte in discontinuità rispetto all'interpretazione tradizionale di Trento: la dottrina circa la sacramentalità dell'episcopato e il ripristino del diaconato come «grado proprio e permanente della gerarchia ecclesiastica» ( $L G 29$ ). D'altra parte, alle tante ricorrenze di minister/ministerium nel decreto fa da pendant un numero non insignificante di richiami alla sua dimensione sa-

19. Episcopus ricorre 334, di cui 24 in SC, 5 in $I M$; 67 in $L G$ (58 in $L G$ III), 11 in Nep; 4 in $O E ; 3$ in $U R ; 138$ in $C D ; 12$ in $O T ; 2$ in $G E ; 1$ in $D V ; 2$ in $A A ; 31$ in $A G ; 32$ in $P O ; 4$ in GS. in $P O$.

20. Presbyter (soprattutto al plurale) ricorre 145 volte, di cui 10 in $L G$ (5 in $L G 28), 122$

21. Diaconus ricorre 14 volte: $S C$ 35. 68. 86; $L G$ 20. 28. 29 (4x). 41. CD 15; DV 25; $A G$ 15. 16 .

22. Il termine ordo ricorre 189 volte, naturalmente con vari significati: quelli che afferiscono al sacramento dell'ordine si trovano prevalentemente in $L G$ (24 volte), $C D$ (8 volte) e $P O$ (18 volte).

23. Il più significativo, per l'importanza del testo nell'architettura del testo, è certamente $L G 10$, dove si pone in relazione sacerdozio comune e sacerdozio ministeriale o gerarchico. 
cerdotale: 31 ricorrenze di sacerdos, 16 di sacerdotalis e 15 di sacerdotium dicono che questo ministero è e rimane comunque caratterizzato in senso sacerdotale.

L'insistenza sui numeri è dettata da una cautela: evitare ogni rischio di interpretare ideologicamente i dati del concilio. Per cogliere le novità del Vaticano II sul ministero ordinato, più che il vocabolario bisogna verificare i singoli interventi che hanno modificato la sintesi dottrinale del concilio di Trento. Un esame diacronico permetterà di seguire lo sviluppo del tema attraverso le sessioni conciliari, cogliendo peraltro un primo processo di recezione interno ai documenti del Vaticano II. Processo che inizia già da Sacrosanctum concilium e concentra gli elementi di novità soprattutto in Lumen gentium.

\section{IL SACERDOZIO DI CRISTO}

Il primo «luogo» obbligato in cui cercare elementi di novità sul ministero ordinato è il capitolo III di Sacrosanctum concilium. Nel quadro delle indicazioni circa la riforma dei sacramenti e dei sacramentali, $S C 76$ dice che «i riti delle ordinazioni siano riveduti quanto alle cerimonie e ai testi», senza specificare oltre $^{24}$. Si trattava di materia già profondamente riformata: Pio XII, con la costituzione apostolica Sacramentum Ordinis $^{25}$, aveva già disposto il superamento della traditio instrumentorum, ripristinando per ogni ordine l'imposizione delle mani accompagnata «dalle parole che determinano l'applicazione di questa materia, con cui in modo univoco vengono significati gli effetti sacramentali» ${ }^{26}$. Sacrosanctum concilium non cita quella costituzione, ma si limita a una richiesta generica, in linea con i principi generali per la riforma e l'incremento della sacra liturgia, esposti nel capitolo I della costituzione.

$\grave{E}$ in tale contesto che si incontra il primo elemento di novità. Elemento in genere passato sotto silenzio, probabilmente perché non utilizza esplicitamente il vocabolario usuale per il sacramento dell'Ordine. Ma quando si spieghi la liturgia come «opera della redenzione umana e della perfetta glorificazione di Dio» (SC 5), è del tutto evidente che la funzione centrale

24. Il paragrafo si limita a dare la possibilità della lingua vernacola per le allocuzioni che precedono l'ordinazione sacerdotale o la consacrazione episcopale, e a concedere che in quest'ultima tutti i vescovi presenti impongano le mani sul consacrando.

25. Pio XII, Costituzione apostolica Sacramentum Ordinis (30 novembre 1947), in AAS 40 (1948), 5-7; DH 3857-3861.

26. DH 3859. 
in quest'opera spetti al Cristo, «unico Mediatore tra Dio e gli uomini» (1Tm 2,5 ). Tale funzione è sacerdotale, come chiarisce il testo spiegando la presenza di Cristo nella liturgia:

Giustamente la liturgia è considerata come l'esercizio della funzione sacerdotale di Gesù Cristo, mediante la quale con segni sensibili viene significata $\mathrm{e}$, in modo proprio a ciascuno, realizzata la santificazione dell'uomo, e viene esercitato dal corpo mistico di Cristo, cioè del capo e dalle sue membra, il culto pubblico integrale (SC 7).

Il fatto di attribuire a Cristo la funzione sacerdotale sembra del tutto ovvio: anche il concilio di Trento parlava di Cristo come Sommo Sacerdote secondo l'ordine di Melchisedech, con la funzione di «condurre ad ogni perfezione coloro che dovevano essere santificati $\gg{ }^{27}$. Ma la differenza tra le due prospettive è abissale: a Trento si sottolineava il sacrificio della croce come atto sacerdotale sommo, attraverso il quale Cristo compie la salvezza; al Vaticano II si enfatizza la presenza attuale di Cristo, morto e risorto, nelle azioni liturgiche, che sono «azioni di Cristo e della Chiesa» (SC 26). Nella logica della liturgia tridentina il centro e il fondamento di tutto è l'efficacia universale del sacrificio di Cristo: perciò la Messa è compresa come ripetizione incruenta del sacrificio compiuto dal Redentore «sull'altare della croce ${ }^{28}$. Nella logica del Vaticano II, invece, il fondamento della liturgia e della vita della Chiesa è «il mistero pasquale della sua beata passione, resurrezione da morte e gloriosa ascensione al cielo, mistero con il quale «morendo ha distrutto la nostra morte e risorgendo ha rinnovato la vita»»» (SC 5).

Sta qui la grande rivoluzione di Sacrosanctum concilium, che elegge l'idea di mistero pasquale, così centrale nel Nuovo Testamento e nei Padri, ad architrave non solo della liturgia, ma della vita della Chiesa ${ }^{29}$. Certo, anche

27. Concilio di Trento, Dottrina e canoni sul sacrificio della Messa, cap. 1: DH 1739. Il Catechismo romano, spiegando il sacerdozio di Cristo, afferma che «è stato Lui che, fornito della somma potestà di dare la grazia di rimettere i peccati, lasciò alla sua Chiesa questa potestà, sia pure limitata nella natura e ristretta nei Sacramenti»: Catechismo romano, parte II, cap. VII, n. 8.

28. Tanto coincidono croce e altare, che le proprietà dell'una sembrano attribuibili all'altro e viceversa. Concilio di Trento, Dottrina e canoni sul sacrificio della Messa, cap. 1: DH 1740 .

29. La distanza tra i due modelli di liturgia - e perciò di sacerdozio - si misura sulla distrazione della liturgia tridentina rispetto al mistero pasquale. Gli elementi che esprimono il mistero pasquale erano tutti presenti nella liturgia tridentina, ma senza nesso tra loro. Per capire quanto l'idea fosse marginale, basta rammentare che la veglia pasquale si celebrava al mattino del sabato santo. 
per Trento il sacerdozio di Cristo «non doveva estinguersi con la morte» $»^{30}$; ma la soluzione è diametralmente opposta a quella indicata dal Vaticano II. Se a Trento Cristo, «proclamandosi sacerdote secondo l'ordine di Melchisedech», nell'ultima cena ha voluto «lasciare alla Chiesa, sua amata Sposa, un sacrificio visibile» da ripetere fino alla fine dei tempi ${ }^{31}$, il Vaticano II dice che «per realizzare un'opera così grande, Cristo è sempre presente alla sua Chiesa» (SC 7). «Semper adest» non dice tanto la presenza di Cristo «nella», ma «alla Chiesa», nella modalità di colui che si fa presente, che viene per agire a favore di qualcuno. Non a caso, dopo aver elencato le forme di presenza nella liturgia, il testo specifica che «in quest'opera così grande Cristo associa sempre a sé la Chiesa, sua sposa amatissima, la quale prega il suo Signore e per mezzo di lui rende culto all'eterno Padre» (SC 7).

Si tratta sempre di un'azione di Cristo a favore «della Chiesa sua amata sposa». Ma diversa è la sua interpretazione: l'una riassume l'esercizio della funzione sacerdotale di Cristo nel sacrificio sulla croce, significato dal sacrificio eucaristico; l'altra dispiega la medesima funzione nel tempo attraverso una presenza continua del Cristo morto e risorto, che alla destra del Padre continua la sua opera mediatrice. E diverso è anche il suo prolungamento nella Chiesa: l'una intende il sacerdos come alter Christus, il quale ripete in modo incruento l'unico sacrificio della croce; l'altra vede chi presiede la celebrazione eucaristica come colui che «agisce in persona Christi capitis», con la funzione di rendere «sacramentalmente» presente - quale «segno e strumento» che lo rende visibile - il Cristo che opera nella celebrazione ${ }^{32}$.

\section{IL SACERDOZIO COMUNE}

Dall'affermazione che «Cristo unisce sempre a sé la Chiesa sua sposa amatissima», per cui a rendere culto al Padre è sempre «il corpo mistico di Cristo, cioè il capo e le sue membra» (SC 7), poteva essere dedotto l'esercizio del sacerdozio comune dei fedeli. Ma Sacrosanctum concilium, nell'intento di guadagnare la «piena, consapevole e attiva partecipazione» dei fedeli alle

30. Concilio di Trento, Dottrina e canoni sul sacrificio della Messa, cap. 1: DH 1740.

31. Concilio di Trento, Dottrina e canoni sul sacrificio della Messa, cap. 1: DH 1740.

32. Il passaggio da uno schema sostitutivo a uno schema ripresentativo è enorme: non averlo chiaramente esplicitato è stato causa di grandi fraintendimenti, che hanno determinato la presa di posizione netta di papa Francesco, intervenuto sulla questione del rito romano «straordinario» con il motu proprio Traditionis custodes sull'uso della liturgia romana anteriore alla riforma del 1970 (16 luglio 2021). Le ragioni dell'intervento sono ampiamente spiegate nella lettera all'episcopato che accompagna le decisioni contenute in questo motu proprio. 
celebrazioni liturgiche (SC 14), non esplicita tale aspetto, che nel documento rimane ancora allo stato embrionale ${ }^{33}$. Per un pieno recupero bisognerà attendere la costituzione dogmatica sulla Chiesa, con gli sviluppi sulla teologia del Popolo di Dio, che hanno determinato la cosiddetta «rivoluzione copernicana» del concilio.

In Lumen gentium, in effetti, il sacerdozio comune occupa un posto privilegiato, prima della trattazione sulla costituzione gerarchica della Chiesa. L'evidenza data al tema non deriva tanto dalla novità delle affermazioni, che di fatto ripetono quanto già aveva affermato il Catechismo romano ${ }^{34}$, quanto dalla nuova collocazione che ricevono nel documento con l'inserimento del capitolo sul Popolo di Dio prima di quello sulla costituzione gerarchica della Chiesa. Quel capitolo, voluto per affermare il principio della pari dignità di tutti i membri del Popolo di Dio in ragione del battesimo, è stato costruito scorporando il paragrafo sul sacerdozio comune dal capitolo sui laici, che nello schema discusso in aula seguiva il capitolo sulla gerarchia. Questa semplice traslazione ha funzionato come un cuneo in grado di rompere l'identificazione tra Chiesa e gerarchia, mettendo fine alla Chiesa come societas inaequalium ${ }^{35}$.

In quel modo è stata ribaltata anche la logica che governava la relazione tra sacerdozio ministeriale e sacerdozio comune come forme di partecipazione al sacerdozio di Cristo: se nello schema dato ai Padri l'enfasi cadeva sul sacerdozio ministeriale, superiore «per essenza e non solo per grado» al sacerdozio universale dei fedeli ${ }^{36}$, nella nuova redazione il sacerdozio co-

33. In SC 6 si cita At 2, 41-47, ricorrente nei testi sul sacerdozio comune, ma dal testo - e dal contesto - si deduce unicamente che «da allora la Chiesa mai tralasciò di riunirsi in assemblea per celebrare il mistero pasquale»; in SC 14 si specifica che la partecipazione piena, consapevole e attiva alle celebrazioni è un diritto che, in forza del battesimo, appartiene al Popolo cristiano, che è «stirpe eletta, sacerdozio regale, nazione santa, Popolo che Dio si è acquistato $(1 \mathrm{Pt} 2,9$; cfr 2, 4-5)». Come a dire che alla partecipazione attiva dei fedeli è presupposta l'idea di sacerdozio comune, senza che venga esplicitata. Dopo secoli di silenzio, un rimando così timido equivale a un mancato sviluppo.

34. Basta mettere in parallelo LG 10 con il Catechismo del concilio di Trento, parte II, cap. VII, n. 23. Al di là dello sforzo di superare la caratterizzazione interiore, tipica di una teologia che riduceva i fedeli a fruitori passivi di un'opera compiuta dal solo sacerdote, i due testi coincidono quanto ai contenuti, evidentemente non quanto alla logica che li ispira e governa.

35. Paradossalmente, il testo più chiaro in tal senso è rimasto nel capitolo IV sui laici: «Unico è il Popolo eletto di Dio...; comune è la dignità dei membri per la loro rigenerazione in Cristo, comune la grazia di adozione filiale, comune la vocazione alla perfezione; non c'è che una sola salvezza, una sola speranza e una carità senza divisioni. Nessuna ineguaglianza quindi in Cristo e nella Chiesa per riguardo alla stirpe o nazione, alla condizione sociale o al sesso (Gal 3,28; cfr. Col 3,11)» (LG 32).

36. Non è qui possibile entrare nella discussione se la differenza tra sacerdozio comune e sacerdozio ministeriale sia «di grado» o «di essenza», secondo la frase di Pio XII citata nel 
mune diventa termine necessario per situare e comprendere il sacerdozio ministeriale nella Chiesa. Dopo aver descritto il sacerdozio comune, $L G 10$ precisa che «il sacerdozio comune dei fedeli e il sacerdozio ministeriale o gerarchico, quantunque differiscano essenzialmente e non solo di grado, sono tuttavia ordinati l'uno all'altro, poiché l'uno e l'altro, ognuno a suo proprio modo, partecipano dell'unico sacerdozio di Cristo».

Alla relazione asimmetrica, che sottendeva una superiorità del sacerdozio ministeriale rispetto al sacerdozio spirituale dei fedeli - ridotto alla stregua di una realtà incoativa e metaforica ${ }^{37}$ - e giustificava la concentrazione di ogni capacità attiva nelle mani dei ministri sacri, il concilio sostituisce una relazione di circolarità, in cui sacerdozio comune e sacerdozio ministeriale sono ordinati l'uno all'altro, in quanto forme distinte di partecipazione all'unico sacerdozio di Cristo. Da questa relazione di complementarietà il sacerdozio ministeriale risulta configurato non più come dignità al di sopra dei fedeli - con la pletora di titoli che continuano ad accompagnare le funzioni dei ministri ordinati -, ma come la forma più radicale di servizio al Popolo sacerdotale. Il concilio, a ben vedere, propone qui una conversione radicale ai «sacri ministri», chiamati a maturare la consapevolezza che esistono non perché detengono un potere, ma perché esiste un Popolo da servire!

È vero che al momento di precisare come l'uno e l'altro sacerdozio partecipino «suo peculiari modo» al sacerdozio di Cristo, la bilancia sembra di nuovo pendere a favore del sacerdozio ministeriale ${ }^{38}$. Ma questo vale se si

testo conciliare. Faccio notare che la prospettiva di Pio XII (e della prima redazione dello schema conciliare) era quella della superiorità costitutiva del sacerdozio ministeriale sul sacerdozio regale: in quella logica, poco importava se la differenza fosse di grado o di essenza, perché il sacerdozio ministeriale risultava comunque superiore. Non più quando si rovesci il rapporto: se la differenza è solo di grado, il sacerdozio regale risulta comunque una forma diminuita di partecipazione al sacerdozio di Cristo. Solo se differiscono «per essenza», il sacerdozio comune assume una sua specificità e rilevanza. Ho provato a sviluppare questi temi in: D. Vitali, «Sacerdozio comune e sacerdozio ministeriale o gerarchico: rilettura di una questione controversa», Rassegna di Teologia 52 (2011) 39-60; «Il sacerdozio comune», Rivista Liturgica, 107 (2020) 101-125; «Il Messale Romano: fonte dell'identità ecclesiale», Rivista Liturgica 107 (2020) 65-82.

37. Nel dibattito in aula emergono questi giudizi: così il card. Caggiano, arcivescovo di Buenos Aires (Acta Synodalia, II/III, 173) e il p. Sepinski, generale dei Minori (Acta Synodalia, II/III, 545).

38. Qui il testo non corregge la struttura che aveva nel primo schema, menzionando il sacerdozio ministeriale prima del sacerdozio comune. Quanto ai contenuti, afferma che «il sacerdote ministeriale, con la potestà sacra di cui è rivestito, forma e dirige il popolo sacerdotale, compie il sacrificio eucaristico in persona Christi e lo offre a Dio a nome di tutto il popolo; da parte loro i fedeli, in virtù del loro sacerdozio regale, concorrono ad offrire l'Eucaristia ed esercitano il loro sacerdozio nel ricevere i sacramenti, nella preghiera e nel ringraziamento, nella testimonianza di una vita santa, nell'abnegazione e nell'operosa carità» ( $L G 10)$. 
legga il sacerdozio sul registro del fare, trasformando sacerdozio comune e sacerdozio ministeriali in funzioni concorrenti. In realtà, le forme privilegiate di esercizio del sacerdozio comune non hanno per soggetto i singoli battezzati, ma la universitas fidelium, il Popolo santo di Dio: lo si vede in $L G 11$, dove il soggetto che esercita il sacerdozio comune nei sacramenti è «la comunità sacerdotale», e in $L G 12$, dove risulta che la forma privilegiata di esercizio della funzione profetica è la totalità dei battezzati, dotata del sensus fidei. Più ancora della questione circa i ministeri laicali, spesso condotta sul registro della rivendicazione, sono queste funzioni ecclesiali a dare la reale misura del sacerdozio comune, rimasto lettera morta nel post-concilio ${ }^{39}$.

\section{LA SACRAMENTALITÀ DELL'EPISCOPATO}

La stretta relazione che il concilio stabilisce tra sacerdozio comune e sacerdozio ministeriale obbliga a ridisegnare l'intero quadro del ministero e dei ministeri nella Chiesa, modificando radicalmente anche la comprensione del sacerdozio ministeriale, non più inteso come una dignità sopra il Popolo di Dio: il guadagno è indubitabile ${ }^{40}$. Tuttavia, al momento di ripensare la questione, bisogna rammentare che il sacerdozio ministeriale rimanda a una realtà complessa, che il Vaticano II ha contribuito a rendere ancora più complessa. In effetti, quando insegna che «con la consacrazione episcopale viene conferita la pienezza del sacramento dell'Ordine, quella cioè che la consuetudine liturgica della Chiesa e la voce dei santi Padri chiama il sommo sacerdozio, la somma del sacro ministero» ( $L G 21)$, determina che in relazione con il sacerdozio comune stiano due ordini: quello dei vescovi e quello dei presbiteri, che sono propriamente ordini sacerdotali.

39. Non è fuori luogo mettere in relazione la mancata recezione del sacerdozio comune con il rifiuto dell'ecclesiologia del Popolo di Dio, attuato quantomeno dal Sinodo straordinario del 1985, a partire dal quale si è imposto dall'alto quell'ecclesiologia di comunione fondata sulla categoria di hierarchica communio. Per una rilettura critica di questo passaggio, mi permetto di rimandare a D. Vitali, «La comunione ecclesiale. Rilievi teologici», in: Gruppo italiano di docenti di diritto canonico (eds.), La comunione nella vita della Chiesa: le prospettive emergenti dal concilio Vaticano II. Glossa, Milano 2015, 3-34. È interessante notare che, a fronte di una infinità di interventi sulla differenza di grado o di essenza tra sacerdozio comune e sacerdozio ministeriale - per farsi un'idea della bibliografia, basta andare a $\mathrm{Ph}$. J. Roy, Bibliographie du Concile Vatican II, Città del Vaticano 2012 -, i testi sul sacerdozio comune non arrivano alla decina: sul tema: J. Jouve Soler, El sacerdocio común de los fieles, elemento necesario de la Eclesiología, Roma 2017.

40. Una reale attivazione del rapporto tra sacerdozio comune e sacerdozio ministeriale sarebbe la via più sicura per indebolire alla radice la malapianta del clericalismo. 
Sta qui il terzo elemento di novità, che, al pari dei due precedenti, ha una forza d'urto impressionante, perché interviene in radice sul quadro dottrinale formulato dalla teologia post-tridentina. In quella visione vigeva l'identificazione del sacerdozio con la potestas ordinis: quanto esulava da quella ricadeva nell'ambito della potestas iurisdictionis. La tesi di scuola era che non si desse per il vescovo una nuova ordinazione - esclusa in ragione del carattere sacerdotale impresso nell'ordinazione presbiterale -, ma solo una consacrazione che lo innalzava alla funzione di governo della comunità ecclesiale. In discontinuità con quella visione, il concilio dice che la consacrazione episcopale conferisce non soltanto le funzioni di insegnare e governare - cioè la potestas iurisdictionis - ma la funzione di santificare, e sostiene, richiamandosi alla Tradizione, che la consacrazione episcopale conferisce la grazia dello Spirito santo e imprime il carattere sacro, per cui «i vescovi, in modo eminente e visibile, svolgono la parte di Cristo stesso, Maestro, Pastore e Sacerdote, e agiscono in Eius persona» ( $L G 21)$.

Molti tendono a rifiutare questa dottrina, perché complicherebbe invece che risolvere i problemi, non solo introducendo una caratterizzazione sacerdotale del vescovo ulteriore e successiva rispetto a quella del presbitero, ma aprendo una quaestio disputata di non poco conto: se il sacerdozio dei presbiteri sia partecipazione del sacerdozio di Cristo o del vescovo. Pur consapevole di tali complicazioni interpretative, il concilio non ha avuto dubbi ad affermare che l'episcopato è sacramento, anzi, la pienezza del sacramento dell'Ordine. Le ragioni che hanno determinato l'aula a questa presa di posizione non riguardano tanto la sacramentaria, quanto l'ecclesiologia. In effetti, la dottrina riprende quasi alla lettera lo schema di constitutio secunda de Ecclesia del Vaticano I ${ }^{41}$, discussa ma non promulgata per la sospensione sine die dei lavori conciliari.

Quello schema di costituzione conteneva la dottrina circa i diritti dei vescovi, che bilanciava la dottrina dei primato affermata nella constitutio prima de Ecclesia ${ }^{42}$. I rischi determinati dalla mancata approvazione si avvertirono in Germania, quando von Bismarck, cancelliere del Regno, intese limitare l'azione della Chiesa con il pretesto che le dichiarazioni del Vaticano I sul primato e l'infallibilità avevano ridotto i vescovi a sudditi di un sovrano assoluto straniero. I vescovi risposero riaffermando la dottrina ufficiale del

41. Concilio Vaticano I, Schema constitutionis dogmaticae secuandae de Ecclesia «Tametsi Deus», in Mansi, 53, 308-317.

42. Concilio Vaticano I, Costitutio dogmatica prima de Ecclesia «Pastor Aeternus» (18 luglio 1870), in DH 3050-3074. 
Vaticano I e, al contempo, sostenendo che l'episcopato, al pari del papato, «è di istituzione divina: anch'esso ha i suoi diritti in virtù di questa istituzione, che il papa non ha né il diritto né il potere di cambiare» ${ }^{43}$. Questa dottrina, ritenuta ormai sicura ${ }^{44}$, entra al concilio: già nel primo schema si afferma che «l'episcopato appartiene senza dubbio al sacramento dell'Ordine, ed è sacerdozio nel grado eminente [praecellenti gradu], quello che la voce dei santi Padri e la consuetudine liturgica della Chiesa chiama il sommo sacerdozio, il vertice del sacro ministero» ${ }^{45}$. Il testo è mantenuto anche nella nuova redazione dello schema de Ecclesia, con la correzione che non si tratta del vertice, ma della pienezza dell'Ordine ${ }^{46}$.

\section{LA RICONFIGURAZIONE DEGLI ORDINI}

La dottrina sulla sacramentalità dell'episcopato ha prodotto una doppia conseguenza: una diretta - e direttamente voluta - sul piano ecclesiologico e una indiretta sul piano sacramentale. In ambito ecclesiologico, se il vescovo ha la pienezza del sacramento ed è portatore del tralcio dell'apostolicità (cfr $L G 20$ ), non è più un vicario e ambasciatore del papa, ma di Cristo stesso, con potestà propria, ordinaria e immediata sul popolo che gli è affidato $(\mathrm{cfr}$ $L G$ 27), e la diocesi che amministra non è una provincia della Chiesa universale, ma «una Chiesa particolare, nella quale è presente e agisce la Chiesa di Cristo una, santa, cattolica e apostolica» (CD 11): in ultima analisi, si giustifica qui l'idea della Chiesa come «il corpo delle Chiese», «nelle quali e a partire dalle quali esiste l'una e unica Chiesa cattolica» ( $L G 23)$.

Ma l'affermazione, così feconda dal punto di vista ecclesiologico, comporta sul piano del ministero il sovvertimento degli equilibri nella gerarchia ecclesiastica, imponendo di ripensare le relazioni tra gli ordini, risolto dalla teologia post-tridentina vincolando gli ordini minori e maggiori al sacerdozio e inquadrando ciò che non rientrava nella potestas ordinis come materia pertinente alla potestas iurisdictionis. In discontinuità con quella visione, il concilio abbandona la scala ascendente degli ordini e recupera la concezione gerarchica del primo millennio, situandosi nella prospettiva della successio-

43. Dichiarazione collettiva dei vescovi tedeschi, in $D H 3115$.

44. Così la relatio al primo schema de Ecclesia in Acta Synodalia, I/IV, 123.

45. Concilio Vaticano II, Schema constitutionis dogmaticae de Ecclesia «Aeternus Unigeniti Patris», cap. III: «De Episcopatu ut supremo gradu sacramenti ordinis et sacerdotio». In Acta Synodalia, I/IV, 23.

46. Concilio Vaticano II, Schema constitutionis dogmaticae de Ecclesia «Lumen Gentium», textus prior, n, 14, in Acta Synodalia, II/I, 233-234. 
ne apostolica, dalla quale deduce la sacramentalità dell'episcopato. Così gli ordini sono ricomposti in unità dinamica dentro l'unico sacramento:

Cristo, consacrato e mandato nel mondo dal Padre (cfr Gv 10,36), ha reso partecipi della sua consacrazione e missione, tramite gli Apostoli, i vescovi loro successori, i quali, a loro volta, hanno legittimamente trasmesso la loro funzione di ministero secondo vari gradi a soggetti diversi nella Chiesa. In tal modo il ministero divinamente istituito viene esercitato in ordini diversi da coloro che già in antico vengono chiamati vescovi, presbiteri, diaconi (LG 28).

A seguire, Lumen gentium ridisegna il rapporto tra vescovi e presbiteri. Per quanto non possiedano «l'apice della dignità pontificale [pontificatus apicem], i presbiteri sono sacerdoti «ad immagine di Cristo sommo sacerdote», «quali veri sacerdoti della Nuova Alleanza», per quanto l'esercizio del loro ministero sia subordinato ai vescovi. Di loro si dice che sono «provvidenziali cooperatori dell'Ordine episcopale, aiuto e strumento» nel servizio al Popolo di Dio. Anzi, «per quanto destinati ad uffici diversi formano un solo presbiterio con il vescovo», per quanto tutti i presbiteri, «in forza della comune sacra ordinazione e missione, sole legati tra loro da intima fraternità».

Il paragrafo successivo è dedicato ai diaconi. Dando seguito alle richieste dei vescovi nei paesi di missione, che vedevano nella restaurazione di questo ordine una via d'uscita alla carenza endemica di preti nelle loro diocesi, l'aula accetta - non senza fatica - di abbandonare l'idea del diaconato come ordine transeunte, riconfigurandolo come «grado proprio e permanente della gerarchia ecclesiastica» ( $L G 29)$. Precisa che si tratta di vero sacramento, ma ne segnala la natura non sacerdotale, in quanto ai diaconi «vengono imposte le mani non ad sacerdotium, sed ad ministerium»; sottolinea che i diaconi sono chiamati a svolgere in comunione con il vescovo e con il presbiterio il loro ministero, che consiste nella «diaconia della liturgia, della parola della carità», dedicandosi soprattutto «ai compiti della carità e dell'amministrazione» ${ }^{47}$.

Il discorso sui diaconi non sarà ripreso da un altro decreto di riforma, come invece accadrà per i vescovi e i presbiteri. Christus Dominus applicherà alla funzione pastorale dei vescovi i principi teologici affermati in Lumen gentium; Presbyterorun ordinis, invece, apporterà un approfondimento notevole al ministero dei presbiteri. Il percorso fatto dal concilio sul tema si può misurare dal cambio dei titoli al decreto: de clericis il primo; de

47. Sull'argomento, mi permetto di rimandare a D. Vitali, Diaconi, che fare? San Paolo, Cinisello B. (Mi) 2019. 
sacerdotibus il secondo; de presbyterorum ministerio et vita il terzo ${ }^{48}$, con il recupero della specificità dell'ordine presbiterale e la sua forte caratterizzazione in senso ministeriale, che costituisce l'elemento di maggior novità del decreto.

\section{I «TRIA MUNERA»}

Non si può chiudere questa rassegna circa le novità conciliari sul ministero ordinato senza riferirsi a un elemento in evidente discontinuità con il quadro precedente: la configurazione del ministero come esercizio dei tria munera. La novità non consiste unicamente nell'assunzione dello schema tripartito delle funzioni di insegnare, santificare, governare, peraltro applicato a tutti i soggetti nella Chiesa $^{49}$, ma nel fatto che questo sostituisce lo schema della duplice potestas: ordinis et iursdictionis.

I sostenitori del ritorno alla duplice potestas ritengono l'adozione dei tria munera una concessione alla teologia del tempo, adducendo come ispirazione le tesi di Yves Congar, che indubbiamente ha recitato una parte da protagonista nella redazione di Lumen gentium $^{50}$. Ma se questo può valere per l'applicazione dello schema a tutti i soggetti del Popolo di Dio, non vale per la potestas sacra, che già dal Vaticano I aveva conosciuto uno slittamento verso la tripartizione delle funzioni. La rigida distinzione di ordine e giurisdizione, in effetti, è stata rotta dal dogma dell'infallibilità del papa: la funzione di magistero, che ricadeva nella potestas iursdictionis, veniva ad assumere una tale importanza da essere considerata come potestas a se stante, distinta da quella di ordine e da quella di giurisdizione. Lo schema constitutionis secundae de Ecclesiae non solo dedica un capitolo al magistero, ma parla esplicitamente della gerarchia come dotata di una potestà divinamente istituita e distinta in tria potestates ${ }^{51}$. Il concilio riprende quella dottrina, preferendo il termine munus: una sola potestas, esercitata in tria munera: docendi, sanctificandi, regendi ${ }^{52}$.

48. Per il raffronto degli schemi: F. Gil Hellín, Concilii Vaticani II synopsis. Presbyterorum Ordinis, Città del Vaticano 1996.

49. Per il Popolo di Dio, $L G$ 10-12; per i vescovi, $L G$ 20. 21. 25-27; $C D$ 12-16; per i presbiteri, $L G$ 28; PO 4-6; si applica analogicamente lo schema anche ai diaconi: $L G 29$.

50. Il rimando è a Y. Congar, Jalons pour une théologie du laïcat, Paris 1953. Bisogna però ricordare che Congar ha lavorato come perito al cap. II di Lumen gentium, non al III.

51. «È da ritenere come dogma della fede cattolica che alcuni ricevano nella Chiesa una potestà di santificare, insegnare e governare, che altri non hanno»: Concilio Vaticano I, Schema constitutionis secundae de Ecclesia «Tametsi Deus», cap. III, Mansi, 53, 310.

52. «Potestas», in X. Ochoa, Index verborum, 387-388. 
Lo schema viene usato anzitutto per il Popolo di Dio, quando, nel capitolo II, si parla del sacerdozio comune come partecipazione alle tre funzioni - sacerdotale, regale e profetica - di Cristo ${ }^{53}$. Il capitolo IV dedica un'intera sezione alle tre funzioni ( $L G 34-36$ ), per dettagliare come «tutti i laici, senza distinzioni, sono chiamati a contribuire quali membra vive all'incremento della Chiesa e alla sua continua edificazione» ( $L G 33)$. Per quanto la sezione costituisca una ripetizione che rischia di indebolire le funzioni del Popolo di Dio quale soggetto attivo, trasferendole sui singoli battezzati, l'insistenza sulla partecipazione alla vita e alla missione della Chiesa costituisce un reale guadagno rispetto all'ecclesiologia preconciliare, che concentrava tutte le funzioni nelle mani della gerarchia. Si possono trovare qui i presupposti per sviluppare il tema dei ministeri nella Chiesa ${ }^{54}$.

Mentre per il Popolo di Dio il concilio parla di partecipazione alle funzioni di Cristo, per i vescovi e i presbiteri parla di esercizio delle tre funzioni, che hanno ricevuto - $\mathrm{i}$ vescovi in modo eminente - nell'ordinazione. Per i vescovi si precisa che, «avendo come collaboratori i presbiteri e i diaconi, hanno assunto il ministero della comunità, e presiedono in nome di Dio il gregge di cui sono pastori, in qualità di maestri di dottrina, di sacerdoti del culto sacro e di ministri del governo» ( $L G 20)$. Si ribadisce che nella consacrazione ricevono le funzioni di santificare, di insegnare e di governare, e in un'ampia sezione si precisano le singole funzioni come forma di esercizio dell'unico ministero episcopale. Stesso schema si adotta per i presbiteri, dei quali si dice che, «in virtù del sacramento dell'Ordine e ad immagine di Cristo, sommo ed eterno sacerdote, sono consacrati per predicare il Vangelo, pascere i fedeli e celebrare il culto divino, quali veri sacerdoti della Nuova Alleanza» ( $L G 28)$.

La scelta di affermare al singolare la sacra potestas, esercitata nei tria munera, è dettata dall'affermazione che nella consacrazione episcopale sono unitamente comunicate le funzioni di santificare, insegnare e governare. Non sono negate la potestas ordinis e la potestas iurisdictionis, ma sono ricomposte - come era nel primo millennio - nel ministero del vescovo, espresso nella capacità di santificare, istruire, governare il popolo a lui affidato.

53. Da notare che il capitolo II non sviluppa la funzione regale; la Commissione teologica rimanda su questo a $L G 36$. Il motivo è probabilmente da leggere nella difficoltà di immaginare una partecipazione del Popolo di Dio alla funzione di governo.

54. Da qui sono partito per sviluppare il tema in «Ministeri e ordine», in: O. Aime et al (eds.), Nuovo Dizionario Teologico Interdisciplinare, Bologna 2020, 524-532. 


\section{CONCLUSIONE APERTA}

Sono questi gli elementi di maggiore novità che si possono recensire nei documenti del Vaticano II. Non si tratta di una riformulazione compiuta e sistematica e gli elementi non sempre risultano collegati tra loro e debitamente armonizzati; spesso dipendono da contesti diversi, in particolare l'ecclesiologia. Ma non è compito di un concilio produrre un manuale sul ministero ordinato. Oltre quei testi il Vaticano II non poteva e non doveva andare: la Chiesa, che «nel corso dei secoli tende costantemente alla pienezza della verità divina, finché in essa giungano a compimento le parole di Dio» ( $D V 8)$, a quello stadio di «comprensione - anzi, di perceptio - tanto delle cose quanto delle parole rivelate» $(D V 8)$ era giunta, e quello ha espresso, rivelando anche la fatica ad integrare e riequilibrare in un quadro compiuto gli elementi di novità che ha proposto ${ }^{55}$. D'altra parte, le affermazioni conciliari sono spesso maturate attraverso un confronto travagliato, spesso concluso con testi compromesso, che dovevano tenere conto di posizioni diverse e in tensione tra loro ${ }^{56}$.

Sarà compito della teologia, a partire degli elementi di novità del concilio, ripensare il ministero ordinato, riscattando quei nuclei tematici dal loro carattere rapsodico e componendoli in una sintesi unitaria e coerente. A tutt'oggi, però, questa sintesi stenta a venire: il processo di recezione post-conciliare fatica a metabolizzare gli elementi di novità emersi al Vaticano II e a integrarli in una visione condivisa, in grado di dare non solo coerenza teorica, ma impulso effettivo a un modello di ministero in linea con il quadro ecclesiologico disegnato dal concilio Vaticano II.

I tanti manuali sul ministero ordinato rivelano una doppia linea di tendenza: quelli che si limitano a un'opera di maquillage, assumendo in uno schema consolidato gli elementi di novità compatibili con il quadro prece-

55. Dire, ad esempio, che i presbiteri sono «provvidenziali cooperatori dell'ordine episcopale» ( $L G 28)$ non è falso, ma non rende la verità del legame tra i presbiteri - anzi, del presbiterio - con il vescovo, principio e fondamento di unità della Chiesa particolare al servizio della quale essi sono posti. Ma era difficile scegliere questa via - comunque affermata dal concilio, sia in $L G 28$ che in $P O 8$ - per due punti di resistenza: quello dei presbiteri religiosi, che non rientrano in questo legame, regolati come sono dal principio dell'esenzione, e quello dei vescovi titolari, i quali, non avendo popolo, non possono essere principio di unità di un presbiterio.

56. A volte il concilio non cerca la composizione, ma lascia le distinte affermazioni, senza cercare la formula di compromesso. In ambito del ministero ordinato, il caso più evidente riguarda il carattere: in $L G 21$ si afferma una impressione del carattere con la consacrazione episcopale, passando sotto silenzio il medesimo effetto nell'ordinazione presbiterale; in $P O 2$ si afferma invece che «i presbiteri, con l'ordinazione, sono insigniti di uno speciale carattere che li configura a Cristo sacerdote, in modo da poter agire in persona di Cristo capo». 
dente; quelli che invece si distaccano - spesso anche polemicamente - da quel quadro e ricercano nel concilio un nuovo modello di ministero. La prima linea di tendenza, orientata alla conservazione, pare dedicarsi a un'opera selettiva, che filtra le novità, riconducendole alla logica del quadro tradizionale o espungendole, quando non siano riconducibili dentro quello schema di pensiero; la seconda, tesa alla ricerca di un nuovo modello, pare più preoccupata di individuare una categoria diversa - alternativa - di sintesi, che di ricercare i nessi interni tra i diversi nuclei di novità, dai quali far emergere un quadro coerente, nuovo e insieme fedele allo sviluppo della dottrina cristiana.

Le due linee di tendenza solo in apparenza sono antitetiche: a ben vedere, ambedue si muovono sul bisogno di costruire un sistema a partire da una categoria di riferimento, che si tratti di sacerdozio o di ministero. Ambedue sono a rischio di ideologizzazione: ampiamente dimostrata la prima, facilmente intuibile la seconda. Per contro, gli elementi di novità che il concilio ha consegnato alla Chiesa domandano che si comprenda il ministero ordinato come unità dinamica dei tre ordini, e che tale unità si imposti a partire dalle relazioni con il vescovo, quale principio di unità della sua Chiesa.

Con questo è anche indicato il criterio che deve regolare lo sviluppo del discorso: la necessaria corrispondenza tra modello di Chiesa e modello di ministero. La forza del modello tridentino dipendeva dalla coincidenza dei due modelli, tradotti nella Chiesa clericale; il superamento di questa, cercato e voluto dal concilio, deve portare verso un modello di ministero che, senza nulla perdere delle ricchezze della Tradizione, corrisponda all'idea della Chiesa come «corpo delle Chiese», «nelle quali e a partire dalle quali esiste l'una e unica Chiesa cattolica» ( $L G 23)$. Qui, il concilio ha offerto quantomeno l'icona di riferimento: come dice $S C 41$, è «nella partecipazione piena e attiva di tutto il Popolo di Dio alle medesime celebrazioni liturgiche, soprattutto alla medesima Eucaristia, nell'unica preghiera intorno all'unico altare, cui presiede il vescovo circondato dal suo presbiterio e dai ministri» che si ha la «praecipua manifestatio Ecclesiae»; anche dal punto di vista del ministero ordinato. 


\section{BIBLIOGRAFIA CITATA}

Congar, Y., Jalons pour une théologie du laïcat, Paris 1953.

Gil Hellín, F., Concilii Vaticani II synopsis. Presbyterorum Ordinis, Città del Vaticano 1996.

Jouve Soler, J., El sacerdocio común de los fieles, elemento necesario de la Eclesiología, Roma 2017.

Ochoa, X., Index verborum Concilii Vaticani II, Roma 1967.

Roy, Ph. J., Bibliographie du Concile Vatican II, Città del Vaticano 2012.

Vitali, D., «Il Messale Romano: fonte dell'identità ecclesiale», Rivista Liturgica 107 (2020) 65-82.

Vitali, D., «Il sacerdozio comune», Rivista Liturgica 107 (2020) 101-125;

Vitali, D., «Ministeri e ordine», in: O. Aime et al (eds.), Nuovo Dizionario Teologico Interdisciplinare, Bologna 2020, 524-532.

Vitali, D., «Sacerdozio comune e sacerdozio ministeriale o gerarchico: rilettura di una questione controversa», Rassegna di Teologia 52 (2011) 39-60;

Vitali, D., Diaconi, che fare?, San Paolo, Cinisello B. (Mi) 2019. 